\title{
Capitalismo y arqueología tecnológica: análisis sobre el uso de dispositivos digitales*
}

\author{
Valeria Yarad Jeada** \\ Recibido: 2020-06-16 • Enviado a pares: 2020-06-13 \\ Aprobado por pares: 2020-07-21 • Aceptado: 2020-08-22 \\ https://doi.org/10.22395/angr.v19n38a6
}

\begin{abstract}
Resumen
La crisis económica mundial que empezó en 2008 ha generado una caída del poder adquisitivo, reducción del consumo y contracción de la economía. Sin embargo, en medio de la crisis, se desarrolla una vorágine tecnológica que va a la par de un nuevo capitalismo mediante el incremento del uso de dispositivos digitales. El objetivo de este trabajo es analizar las nuevas claves de un capitalismo tecnológico generado por la democratización del consumo de bienes y servicios digitales y una arqueología tecnológica con periodos de obsolescencia cada vez más cortos. Es relevante la comprensión de este tipo de cambios, ya que permiten observar la relación entre el hombre y la tecnología y los usos que hacen de ella, con un enfoque en mejorar su calidad de vida y cerrar brechas como lo es la digital. Este trabajo usa una metodología del tipo documental de datos secundarios a nivel mundial sobre consumo tecnológico y su uso a través de datos provenientes de ITU, Cepal, Eurostat y otros. Además, se realizó un análisis temporal del Smartphone desde el 2008 para establecer el crecimiento de su uso, teniendo en cuenta que ésta una de las tecnologías más utilizadas. Entre los hallazgos más notorios se evidencia que más del $75 \%$ de la población a nivel mundial cuenta con un teléfono móvil, concluyendo que esta tecnología facilita la gestión de la vida cotidiana en todos los aspectos: laboral, familiar, educativo, social, etc., es así que el Smartphone se convierte en una "navaja suiza" que facilita el diario vivir del ser humano.
\end{abstract}

Palabras clave: sistema económico; industria de la comunicación; consumidor; cambio tecnológico; sociedad de la información; análisis de datos.

Este trabajo se enmarca en parte del trabajo realizado desde hace algunos años relacionado con la tesis doctoral: Procesos de uso y consumo de nuevas tecnologías digitales: un análisis específico sobre las prácticas en torno a dispositivos de reproducción móvil digital de quien presenta este manuscrito. Esta tesis doctoral fue defendida dentro del programa de Sociología y Antropología de la Universidad Complutense de Madrid, en la Facultad de Sociología y Ciencias Políticas en el año 2017. La tesis completa se encuentra en el siguiente repositorio institucional: https://eprints.ucm.es/47434/1/T39873.pdf.

. Doctora en Sociología y Antropología y máster en Metodología de la Investigación en Ciencias Sociales por la Universidad Complutense de Madrid, licenciada en Comunicación Social por la Universidad Central del Ecuador. Investigadora independiente y Senior Data Analyst en Alto Data Analytics. Quito, Ecuador. Correo electrónico: vale.yarad@gmail.com. Orcid: https://orcid.org/0000-0002-5040-4324 


\title{
Capitalism and Technological Archaeology: Analysis on the Use of Digital Devices
}

\begin{abstract}
The global economic crisis that started in 2008 has generated a decrease in purchasing power, reduced consumption and a contraction in the economy. However, in the midst of the crisis, a technological maelstrom develops, which goes hand in hand with a new capitalism through the increase in the use of digital devices. The objective of this work is to analyze the new keys to a technological capitalism generated by the democratization of the consumption of digital goods and services and a technological archeology with increasingly shorter periods of obsolescence. The understanding of these types of changes is relevant since they allow us to observe the relationship between man and technology and its usesthe uses made of it, with a focus on improving their quality of life and closing gaps, such as the digital one. This work uses a documentary type methodology of secondary worldwide data on technological consumption and its use through data from ITU, Cepal, Eurostat and others. In addition, a temporal analysis of the Smartphone was carried out since 2008 to establish its growth, since it is one of the most widely used technologies. Among the most notorious findings, it is evidenced that more than 75 \% of the world population has a mobile phone, concluding that this technology facilitates the management of daily life in all aspects: work, family, educational, social, etc. This is how the Smartphone becomes a "Swiss Army knife" that facilitates the daily life of human beings.

Keywords: economic systems; communication industry; consumer; technological change, information society; data analysis.
\end{abstract}




\section{Introducción}

El ser humano vive y convive bajo un nuevo paradigma, el tecnológico. Boiko (2019) habla sobre la "era dorada de la tecnología", cuyo proceso viene desde la Segunda Guerra Mundial y ha generado un cambio en los modelos de producción y apropiación de bienes y servicios, reflejando en muchos casos una discrepancia entre el desarrollo tecnológico y el crecimiento económico. Los bienes lujosos, distintivos, pertenecientes a las altas clases sociales, esos que representaban un estatus (Bourdieu, 1988; Bourdieu 2003; Simmel, 1988; Simmel, 2002), pasan por un proceso de vulgarización, ya que su compra y consumo son ya algo cotidiano para casi todos los sujetos, por ejemplo, el teléfono inteligente o Smartphone, las tabletas o los iPod, fueron en su lanzamiento bienes suntuosos, poco accesibles a la población promedio. No obstante, en la actualidad son parte del consumo masivo y no son exclusivos de las clases altas; convirtiéndose en una herramienta para la comunicación e incluso para el trabajo (Goggin y Hjorth, 2009; Goggin, 2006; Katz y Aakhus, 2002). Son significativos los cambios experimentados desde la era de la producción en masa o fordismo (Alonso, 2007) a una sociedad del conocimiento (Carnoy y Castells, 2001; Castells, 2007) en donde las fronteras entre producción y consumo son más tenues gracias a un nuevo consumidor más activo, dinámico y exigente (Ritzer y Jurgenson, 2010; Toffler y Martín, 1990).

Es así que a la par de este nuevo capitalismo (Fisher, 2010; Gee, 2018; Schiller, 2014) centrado en la innovación, el conocimiento y el uso de herramientas y dispositivos tecnológicos, las estrategias de marketing y consumo son cambiantes, incluso gracias a la Internet, el comercio electrónico (e-commerce) y las redes sociales, las pequeñas, medianas y grandes compañías deben crear nuevas palancas comerciales para atraer a esos consumidores.

El endeudamiento va a ser una pieza clave para la generación del consumo (Lipovetsky, 2007), siendo el consumo de bienes tecnológicos uno de los más atractivos a la hora de adquirir una deuda. En la mayoría de países de Europa (Marchetti y Martínez Carrascal, 2013; Martínez Carrascal y Del Río, 2004) y Latinoamérica (Baker, 2009), los grandes almacenes ofrecen a sus clientes facilidades de pago, algunos con o sin intereses, lo mismo las tarjetas de crédito y los bancos, esto promueve que la economía familiar no tenga un impacto tan directo a corto, sino a mediano y a largo plazo, tomando en cuenta los intereses, las tasas de amortización, entre otros aspectos.

También las compañías de telefonía móvil publicitan distintas ofertas para acercar este consumo a sus potenciales consumidores, los paquetes con Internet móvil y minutos que incluyen teléfono inteligente o tableta son los más promocionados por 
las empresas, al igual que aquellas opciones de renovación del móvil que consisten en la entrega de su "antiguo" dispositivo como forma de pago.

En esta revisión no se puede dejar de lado a las plataformas de compra online como lo son Amazon, eBay, Mercado Libre en Latinoamérica, OLX, Alibaba (Oriente) y Alixpress y Wish (Mundial), entre otras, cuyas empresas han desarrollado estrategias comerciales y de comunicación que atraen a cientos de compradores por las ventajas competitivas en torno a sus precios, la facilidad de comprar sin la necesidad de salir y en algunos casos, como en los de Amazon o eBay, la garantía de los productos que se ofrecen en esas plataformas y su servicio al cliente (Betz, 2002; Kimble y Bourdon, 2013; Ritala, Golnam, y Wegmann, 2014) son un valor agregado para los compradores. Esto ha generado que muchos clientes opten por adquirir dispositivos digitales por medio de estas páginas, ya que comercializan marcas populares como Samsung, Apple, Huawei, etc.

A todo ello, cabe añadir, que este nuevo tipo de consumo es parte de la nueva era de la globalización. Mediante la liberación de los mercados, la economía trasnacional y la reducción de precios en algunos bienes de consumo masivo (Beck, 1998; Beck, Lash, y Giddens, 1997), estos nuevos procesos estimulan la compra. Sin embargo, con eso no se pretende decir que el comprador responde siempre a los estímulos, es más, los consumidores del siglo XXI son cada vez más reflexivos, más proactivos, los llamados prosumidores o prosumers (Kotler, 2010; Ritzer, 2000; Toffler y Martín, 1990) participan de forma activa en los procesos de producción o desarrollo de producto o marca, los influencers establecen a través de las redes y medios sociales las modas y las necesidades de los usuarios para que el productor diseñe y elabore esos bienes y servicios más adaptados a los clientes.

Es así que cada vez más paulatinamente se lanzan al mercado productos tecnológicos, generando una alta expectación entre los usuarios. Un ejemplo de ello, eran las tan ansiadas presentaciones del anterior presidente de Apple, el fallecido Steve Jobs, en las que mediante la gestión escénica y el desarrollo de narrativas (Sharma y Grant, 2011), atraía a millones de posibles compradores y la Internet se llenaba de foros y comentarios relacionados al nuevo producto que recién lanzado, creando así comunidades virtualescon análisis profundos del nuevo producto, los pro y los contras y lo que le diferencia con la anterior versión. Cabe destacar que previo al lanzamiento de estos nuevos teléfonos inteligentes, como son el iPhone o los deSamsung, los medios de comunicación, blogs y redes empiezan a especular sobre las nuevas funcionalidades, es así que los usuarios revisan previamente esa información y sopesan la compra del producto. 
Dada la brevedad de estos lanzamientos, se crea una competencia entre marcas apuntando a nuevas estrategias publicitarias y de comunicación, en una sociedad interconectada y más digital (Soengas et al., 2015) en donde los mensajes tienen que adaptarse a esos nuevos públicos, que son cambiantes, más exigentes y participativos. Las marcas buscan atrapar la atención de los consumidores, quienes día a día observan y escuchan cientos de anuncios tanto en medios tradicionales como en Internet, en palabras de Bermejo (2013)una neopublicidad que utiliza nuevas retóricas, en la que prima la naturalidad, es decir, el "hacer publicidad como si no se hiciera publicidad" (p. 109). Es por eso, que las marcas se han apoyado en influencers, o youtubers o incluso en personas que no son conocidas y que han llegado a serlo gracias a la promoción de alguna de sus marcas.

En lo que se refiere a nuevas formas de consumo, este se convierte en un entramado en el que entran en juego estrategias de marketing, publicidad, redes sociales, moda, tendencias y otros aspectos. Además, el propio mercado va a convertir en obsoletos ciertos artículos, debido a que el ser humano buscará nuevas funcionalidades que le permitan gestionar su cotidianidad (trabajo, estudios, familia, etc.) (Gerard Goggin y Hjorth, 2009; Katz y Aakhus, 2002).

Uno de los ejemplos más notables es WhatsApp, cuya aplicación de mensajería instantánea permite establecer una comunicación directa entre personas y grupos con distintos fines como socialización a partir de mensajes, memes, bromas, ví deos, música, etc., (Ballesteros Doncel, 2016; O’Hara, Massimi, Harper, Rubens, y Morris, 2014; Rubio Romero y Perlado Lamo de Espinosa, 2015), educación (Bouhnik y Deshen, 2014) e incluso para la medicina (Johnston et al., 2015).Esta aplicación, tan popular en los últimos años, dejó de funcionar en algunos sistemas operativos, desde el 31 de diciembre de 2019 no estará disponible para teléfonos Windows, Android 2.3.7 o anterior, y versiones anteriores a iOS 8 para iPhone; en el caso de Android o iPhone es solucionable, sin embargo, en el caso de Windows Phone no, ya que la aplicación no estará disponible en su tienda virtual. Windows Phone se lanzó en 2010, su última versión fue el Windows Phone 8.1 de 2014, si se analiza con mayor detenimiento apenas han pasado 6 años, sin embargo, en el nuevo capitalismo, es una tecnología ya obsoleta que no ofrece las funcionalidades de los móviles que se lanzan al mercado cada año, aunque en temas de hardware el dispositivo mantenga sus piezas intactas y funcionales, las aplicaciones ya no se ajustan a las necesidades de los usuarios porque con el avance de las comunicaciones y de los mercados las dinámicas son cambiantes y para enfrentarse a este nuevo capitalismo tecnocientífico los seres humanos deben adaptar sus destrezas a estas nuevas herramientas y tecnologías. 
En este artículo se denominará a este proceso como una "arqueología tecnológica". En el inicio de la era fordista, los artículos tendían a durar más tiempo y eran símbolo de estatus socioeconómico (Bauman, 2006; Lipovetsky, 2007), mientras que desde inicios del siglo XXI el cambio de artículos, especialmente tecnológicos, se acorta cada vez más, siendo viejos con apenas poco tiempo de uso.

Este concepto se pondrá en discusión mediante el análisis de datos de uso de tecnología en un contexto mundial y el desarrollo de un análisis temporal sobre dispositivos digitales.

\section{Metodología}

Para el desarrollo de este artículo se han tomado en cuenta dos metodologías que permitan responder a la pregunta por silos seres humanos están sujetos a un capitalismo tecnocientífico que ha generado una obsolescencia de los dispositivos tecnológicos existentes.

Para ello, se ha utilizado un análisis documental de fuentes secundarias que permitirán entender en profundidad el contexto. Esta técnica permite la revisión completa de distintos documentos oficiales y provenientes de organizaciones de la sociedad civil que investigan este tipo de fenómenos. Si bien es cierto que en temas tecnológicos existe un vaivén de datos e informaciones que muchas veces no son precisos, ello deja una puerta abierta a la difusión de cifras que no son las reales. Márquez (2006) señala que estos datos que han sido generados en distintas investigaciones y ámbitos permiten que otros investigadores los utilicen para otras finalidades, es decir, pueden ser adaptados al estudio que se realiza. En el campo de la tecnología son cada vez más frecuentes el uso de este tipo de fuentes para el análisis del acceso, consumo y uso de dispositivos digitales, cabe destacar el Informe de la Sociedad de la Información desarrollado por Fundación Telefónica (2019), así como los de Calderón (2019) quien analiza las brechas digitales en España o de Tsetsi y Rains (2017) quienes investigan por medio de datos secundarios el uso del Internet por medio del teléfono inteligente en Estados Unidos.

Para este estudio se han tomado en cuenta fuentes como la International Telecommunications Union (ITU), La Oficina Europea de Estadística (Eurostat), la Comisión Económica para América Latina y el Caribe-Cepal, entre otras. Mediante esta recopilación de información se puede tener una visión macro sobre el uso de tecnologías, dispositivos y la tendencia en el mercado.

Posteriormente, y aprovechando la recopilación de datos cuantitativos, se desarrollará un análisis temporal sobre el crecimiento y consumo de tecnologías 
digitales desde el año 2008 que empezó la crisis económica a escalamundial. En éste se observarán los dispositivos más utilizados y cuáles son las principales estrategias de marketing que se han llevado a cabo para promocionarlos.

\section{Resultados}

\section{Breve Radiografía Sobre Usos y Consumos de Tecnología en la Era Actual}

Para empezar este apartado, se va a citar a uno de los referentes en el campo de la investigación técnica, Lewis Mumford (1971; 1969), quien analizaba la máquina y su importancia en las civilizaciones antiguas y modernas, además de los mitos que rodean el avance tecnológico, mediante posiciones del tipo apocalípticas que incluso hablaban de robots dominando el mundo:

Durante el último siglo, la máquina automática o semiautomática ha llegado a desempeñar un gran papel en nuestra rutina diaria y hemos llegado a desempeñar un gran papel en nuestra rutina diaria; y hemos llegado a atribuir al instrumento físico en sí mismo el conjunto de costumbres y métodos que lo crearon y lo acompañaron. (Mumford, 1971, p.2.)

Es así, que el ser humano es quien adapta estas tecnologías a su diario vivir y no viceversa, la tecnología se asocia a las necesidades y desafíos de la sociedad, dejando de lado esa visión tecnófoba en la que las máquinas dominarán al ser humano, lo que se refleja en algunas películas y series de ciencia ficción.

Los datos apuntan a un crecimiento acelerado en cuanto al uso y consumo de tecnologías móviles como es el teléfono, en detrimento de la telefonía fija (International Telecommunication Union, 2018). Desde 2007, la ITU realiza la medición del uso de tecnología y en el último reporte, en el que participaron 192 países, se afirma que gracias a la virtualidad y a la flexibilidad del teléfono móvil (Katz, 2006; Katz y Aakhus, 2002) este se ha convertido en la tecnología con mayor apogeo entre las demás, a esto se suma que el precio de los celulares móviles han seguido una tendencia decreciente en la última década (International Telecommunication Union, 2018). Cabe señalar que, la telefonía móvil ha permitido acercar socialmente a poblaciones aisladas y facilitar sus labores productivas (Goggin y Hjorth, 2009).

También mediante el uso de apps se han generado las condiciones necesarias para el cambio de ciertos patrones de consumo, un ejemplo de ellos son plataformas como Uber, Deliveroo, Glovo, entre otras, quienes han sido analizadas desde la economía circular y la precariedad e inseguridad laboral de sus "empleados" (Fleming, 2017). 
Es así que el teléfono móvil no solo tiene una alta penetración en el entorno global, un 76,4 \% de la población posee un teléfono móvil, sino que el aumento de suscripciones en banda ancha móvil y de paquetes de minutos va en crecimiento. En la siguiente gráfica se observa que hay más suscripciones de celulares móviles que personas en el planeta, esto se debe a que muchas personas tienen más de una suscripción, para aprovechar los planes competitivos de voz o datos de más de un operador, o porque pueden tener una suscripción profesional y otra personal (International Telecommunication Union, 2018, p. 5).

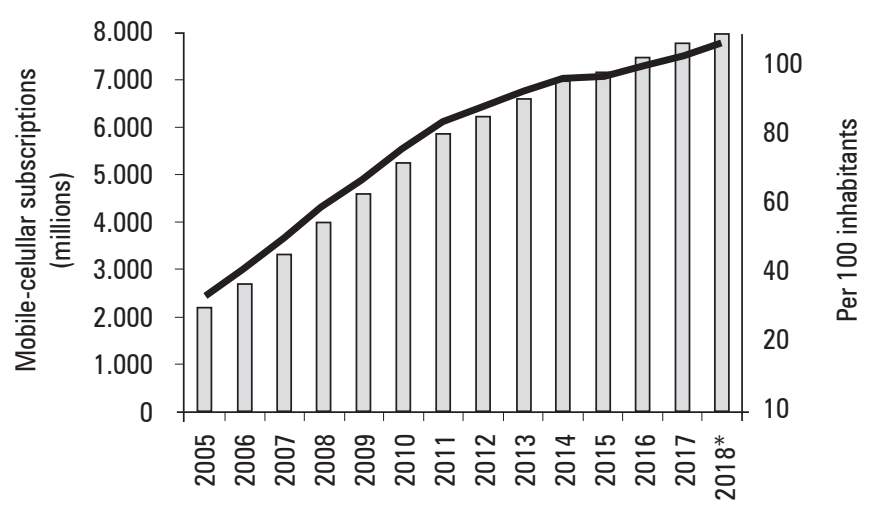

$\square$ Subscriptions (in millions) —Per 100 inhabitants

Figura 1. Suscripción global de móviles, total y por cada 100 habitantes entre 2005-2018.

Fuente: International Telecommunications Union, 2018.

En lo que respecta a la suscripción a servicio móvil por tipo de países, la ITU (2018) hace una clasificación entre países desarrollados, en vías de desarrollo y poco desarrollados, o también llamados Least Developed Countries (LDC) y que se sitúan mayoritariamente en África. Los datos dejan los siguientes resultados: en el caso de los países desarrollados existen 110 suscripciones por cada 100 habitantes, en los países en vías de desarrollo 80 suscripciones por cada 100 habitantes y en los LDC un total de 50 suscripciones por cada 100 habitantes, lo que deja entrever una marcada brecha digital en torno al desarrollo y al ingreso per cápita por países.

El mercado de las aplicaciones de móvil con un dominio en las tiendas virtuales App Store y Google Play también ha representado un importante nicho en las economías a nivel internacional, con un ingreso de 30,5 billones de dólares que son las ganancias de las más de 57 billones de aplicaciones móviles desarrolladas y descargadas en 2017 en todo el mundo (International Telecommunication Union, 2018). 
Todo este ascenso en el uso y consumo de tecnologías móviles se genera en el marco de la bajada de precios de este tipo de dispositivos, que pasan a ser considerados de uso cotidiano para la gestión de la vida diaria. Aunque en este sentido cabe hacer una aclaración, y es que en el mercado existen dispositivos que todavía manejan un alto rango de precios, por encima de los $\$ 600$ y hasta los $\$ 1.200$ dólares aquellos de marcas distintivas (Apple o Samsung). No obstante, algunas empresas nuevas de tecnología han empezado a fabricar dispositivos móviles con precios más bajos y con funcionalidades que permiten la comunicación, uso de aplicaciones (imágenes, música, aprendizaje, etc.) y el acceso a Internet. Es así que en base a los datos de la ITU (2018), los precios de los celulares móviles mantuvieron una tendencia a la baja constante en el período 2008-2017 en función al uso masivo de la banda ancha móvil que se ha disparado en los últimos cinco años en consonancia con la disminución del precio de los planes de telefonía móvil que incluyen datos, minutos y mensajes de texto.

Asimismo, según el Eurostat (2019), organismo encargado de agrupar los datos estadísticos de los países que conforman la Unión Europea, un 73 \% de los europeos se conectan a Internet por medio de su teléfono móvil con un incremento del 54 \% desde el año 2011, siendo uno de los mayores avances en términos de acceso a estas tecnologías por medio de este tipo de dispositivos. No es de extrañarse que los países con mayor uso de esta tecnología a través del móvil sean Suecia (92\%), Noruega (91 \%), Países Bajos (88 \%), Dinamarca (87 \%) y España (86\%), pese a que España fue una de las naciones más afectadas por la crisis económica de 2008, lo que puede resultar paradójico, debido a que la recesión no disminuyó el uso y consumo de este tipo de herramientas.

La Comisión Europea mide entre otros aspectos el "[The] Digital Economy and Society Index" (2019) y dentro d estos evalúa algunos como conectividad, capital humano, uso de servicios de Internet, integración a la tecnología digital y servicios públicos digitales. Los resultados apuntan a que Finlandia, Suecia, Países Bajos y Dinamarca obtuvieron las calificaciones más altas de este índice en 2019 y se encuentran entre los líderes mundiales en digitalización. A estos países les siguen Reino Unido, Luxemburgo, Irlanda, Estonia y Bélgica. Sin embargo, algunos otros países aún tienen un largo camino por recorrer. Estas cifras sugieren que las naciones más desarrolladas, aquellas que apuestan por la inversión en Investigación, Desarrollo e Innovación [ID+i] son las que mejores índices presentan y es porque además muchos de sus servicios se ofrecen mediante este tipo de herramientas que buscan incrementar la productividad y la calidad de vida de sus habitantes, siendo parte de ese llamado primer mundo o naciones desarrolladas, en donde los procesos innovadores van a ir a la par con el desarrollo socioeconómico de sus ciudadanos. 


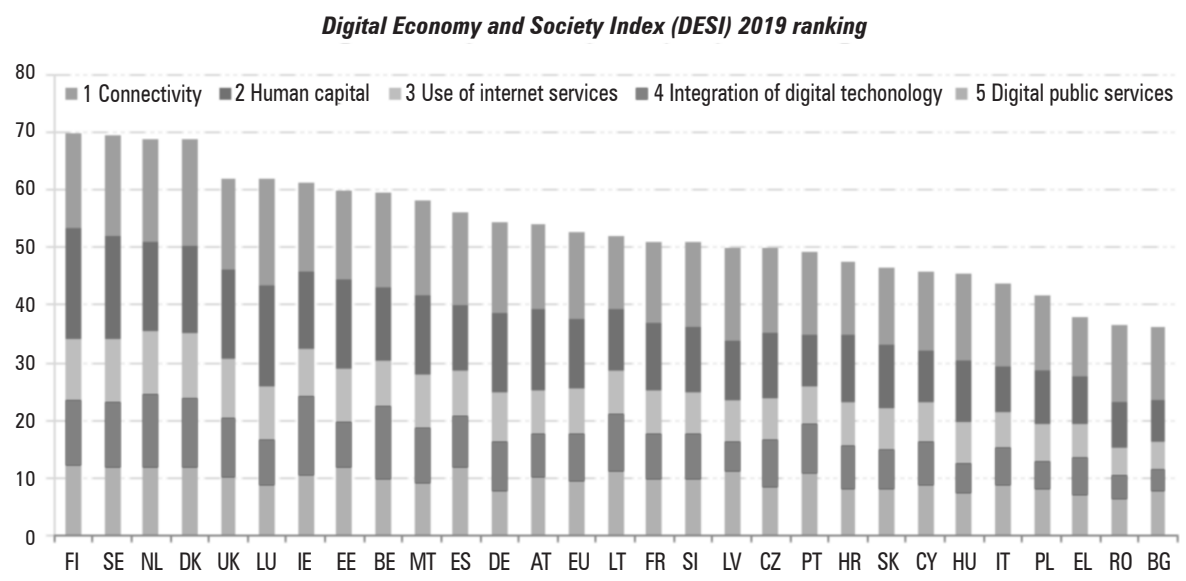

Figura 2. Índice DESI (2019)

Fuente: European Commision, 2019.

En particular se analizará a breves rasgos el tercer indicador relacionado con la práctica de actividades como buscar contenido online (música, videos, juegos, etc.), video llamadas, compras online y transacciones bancarias, las cuales han subido en un $3 \%$ entre 2017 a 2018. Cabe destacar que el mayor incremento del uso de Internet es en video on demand (Netflix, HBO, Amazon Prime, etc.) con un aumento del $21 \%$ en 2016 y del 31 \% en 2018 (European Commision, 2019), teniendo en cuenta que una de las ventajas de estas plataformas es que el usuario puede conectarse desde distintos dispositivos sean estos móviles o fijos.

Otra de las claves que ofrece este índice es sobre las compras online, un $64 \%$ de los europeos han utilizado Internet para comprar algún artículo, frente a un $21 \%$ que lo han utilizado para vender algo. Las categorías de compra más populares fueron ropa y artículos deportivos (64\%), viajes y turismo (53\%), artículos del hogar (45\%), tickets para eventos (39\%), libros, revistas y periódicos (32\%), servicios de telecomunicaciones (20\%), hardware (17\%), entre otros (European Commision, 2019).

En función de esta revisión, la Unión Europea busca la creación de un mercado único digital (Fundación Telefónica, 2019) que además de garantizar el acceso a las tecnologías permita agilizar los procesos en el ámbito de la administración pública y privada y que genere sinergias y marcos de confianza para el correcto uso de las tecnologías.

En el otro lado del mundo se puede ver a Latinoamérica. La Comisión Económica para América Latina y El Caribe, hace una comparativa anual desde 2000 a 2014 del total de subscriptores a teléfonos móviles por cada cien habitantes. En apenas 
catorce años se experimenta un crecimiento exponencial de este tipo de tecnología con más de un 930 \% en la región, se pasa de 12,10 suscripciones de móviles por cada cien habitantes en 2000 a 112,53 líneas por cada cien habitantes en 2014 (Cepal, 2014).

Los países con mayor número de líneas telefónicas móviles por cada cien habitantes son: Anguila $(179,81)$, Surinam $(170,57)$ Islas Vírgenes Británicas $(169,56)$ Uruguay (160,8), Argentina (158,74), Panamá (158,05), Islas Caimán (153,75), Trinidad y Tobago (147,34), El Salvador (144,03), Costa Rica $(143,83)$, Brasil $(133,26)$ y Chile $(133,26)$ (Cepal, 2014). Se observa que la penetración del móvil se da mayoritariamente en islas y archipiélagos del Caribe, así como en los países más industrializados y con mayor índice de desarrollo del Cono Sur.

Por el contrario, el ranking de los países con menor número de líneas por cada cien habitantes es: Cuba $(22,48)$, Belice $(50,41)$, Haití $(64,71)$, República Dominicana $(78,86)$, México $(82,54)$ y Puerto Rico $(87,11)$ (Cepal, 2014). Un resultado algo sorprendente es el de México, ya que es uno de los países más grandes de la región, pero además su cercanía con Estado Unidos puede ser un indicador de mayor intercambio en lo tecnológico, sin embargo, se observa que no todos los mexicanos utilizan el móvil, eso puede deberse a distintos factores como el de la ruralidad, el acceso a estos servicios, la edad, el nivel educativo, entre otros.

Organismos privados, como es la Global System for Mobile Communication (GSMA en sus siglas en inglés), que representa los intereses de los operadores de telefonía móvil a nivel mundial, señala que la tasa de penetración del teléfono móvil en Latinoamérica es del 67 \% hasta 2018 y estima que en 2025 esta tasa aumente al $73 \%$, a mediados de 2019 se estima que hay 422 millones de suscriptores móviles únicos en la región (GSMA, 2019).

Cuando se habla de los tipos de usos que hacen los latinoamericanos, se observa que el móvil y las tabletas además de ser herramientas de comunicación sirven para el entretenimiento y la búsqueda de información en Internet. Se puede observar que de los 422 millones de suscriptores únicos que reporta GSMA (2019) 326 millones utilizan Internet móvil. El reporte también señala que la alta tasa de adopción de este tipo de tecnologías ayuda al cumplimiento de los Objetivos de Desarrollo Sostenible, porque mejora el acceso a la educación y los índices de igualdad de género, con iniciativas que permiten que cada vez más mujeres incursionen en ramas tecnológico-científicas (GSMA, 2019). Así las cosas, es posible evidenciar que pese a las crisis económicas las herramientas tecnológicas como el móvil inteligente no han frenado su crecimiento en la población, ya que son parte de las cotidianidades en un mundo cada vez más digital, dinámico, globalizado y cambiante. 
La mayoría de las naciones han vivido desde hace más de un siglo inmersos en el sistema capitalista (Lipovetsky, 2007), que ha tenido múltiple facetas como es el fordismo, el toyotismo, el imperialismo y ahora la sociedad de la información (Castells, 1997), un capitalismo centrado en otros procesos que van más allá de la máquina, característico de la era del conocimiento y de la innovación, en donde los productos y servicios son cada vez más especializados, pero a su vez son bienes de difusión masiva, en cuyo sistema la obsolescencia será cada vez más acelerada generando además un impacto en la vida de los seres humanos, su entorno e incluso el medio ambiente.

En el siguiente apartado y para cumplir con los objetivos de este trabajo se realizará una revisión temporal desde 2008 del lanzamiento de equipos tecnológicos como el teléfono móvil inteligente y las tabletas hasta el 2020. Se elige este período de tiempo en función de que ese año estalló la crisis económica mundial, la cual afectó especialmente a países del sur de Europa y a Estados Unidos, por lo cual, esta información busca generar una aproximación entre la relación crisisconsumo tecnológico, y se ha traído la cronología hasta 2020 dado que este último año se ha mostrado atípico en lo relacionado al consumo debido a la crisis sanitaria ocasionada por el nuevo Coronavirus Sars-CoV-2.

\section{Análisis Temporal de Lanzamiento de Dispositivos Digitales desde 2008 hasta inicios de 2020}

En el resumen y la introducción se hizo mención a un concepto denominado "arqueología tecnológica", este término se relaciona con ese proceso en el cual una tecnología pese a no ser tan anticuada y tener pocos años en el mercado pasa a ser una reliquia debido a esa vorágine innovadora por la cual cada año se lanzan cientos de productos al mercado que atraen a millones de consumidores, quienes dejan en desuso su anterior dispositivo, puesto que no presenta las nuevas características que tiene el nuevo. El concepto de arqueología tecnológica no ha sido trabajado desde el punto de vista del consumo y la innovación técnico-científica, es así que la autora de este trabajo lo ha propuesto desde su tesis doctoral en 2017 defendida en la Universidad Complutense de Madrid, dentro del programa del doctorado en Sociología y Antropología, en donde pudo observar que los lanzamientos, en lo que respecta a dispositivos digitales, brindaban cada año más y mejores funcionalidades, creando pequeñas brechas entre lo viejo y lo nuevo. En el fordismo (Alonso, 2004), los períodos en los que se ofrecían nuevos productos y servicios eran más prolongados, entre otras cosas, porque la economía estaba más centrada en un tipo de producción más centrado en bienes y servicios del tipo mecánico (autos, electrodomésticos, etc.) y que no tenían la función de ser inteligentes y digitales; por el contrario, el siglo XXI busca esa digitalización que marcha a la par de lo global, 
de una nueva economía y nuevas formas de comunicación, es así que el ser humano va a adaptarse a estos cambios y para ello la tecnología va a ser una herramienta fundamental de inclusión al nuevo mundo globalizado digital.

En 2008 ya existía el teléfono móvil inteligente, cuyos teléfonos inteligentes integran aplicaciones y funcionalidades, convirtiéndose en una especie de "navaja suiza" que permite comunicarse, navegar, entretenerse, etc. El Blackberry es uno de los primeros dispositivos que cumplen con esas características, sin embargo, el iPhone es el referente por excelencia del primer teléfono inteligente, ya que inserta más funcionalidades que el Blackberry, además de su interfaz y su tactilidad (Yarad Jeada, 2017).

El lanzamiento del primer iPhone en 2007 marca el inicio de la carrera por el mejor dispositivo. En ese entonces, el sistema operativo Android de Google, Android 1.0 en versión Beta es instalado en pocos dispositivos como el HTC y el T-Mobile G1, mientras tanto, marcas como Sony, Samsung, Nokia y Motorola contaban con teléfonos que tenían algunas características multimedia, pero que aún no podían ser considerados como Smartphone, debido a las funcionalidades limitadas que ofrecían.

En 2008, el iPhone se convierte en uno de los móviles más vendidos en casi todo el mundo y es entonces cuando la industria tecnológica ve en este fenómeno una oportunidad; es así como, en 2009, Samsung lanza al mercado su primer móvil inteligente con Android, convirtiéndose así en la competencia más directa de Apple en el mercado de dispositivos móviles. El primer Samsung Galaxy S fue uno de los móviles más populares a escala internacional.

En 2010, mientras Apple y Samsung se repartían la mayor parte de la cuota del mercado de móviles inteligentes, Microsoft ofrece al mercado el sistema operativo Windows Phone 7 disponible para Dell, HTC y LG, no obstante, es en 2012 cuando la Microsoft compra la empresa Nokia, que es cuando empieza a comercializar el Nokia Lumia, el cual contaba con la tercera versión del sistema operativo, el Windows Phone 7.8. A la par, Apple lanza su primera tableta, el iPad, posteriormente Samsung presenta la Samsung Galaxy Tab, lo que llevaría a ambas empresas a múltiples arbitrajes legales por temas de propiedad intelectual.

El mundo de la tecnología no para y esto se hace evidente de forma anual en el Mobile World Congress realizado en Barcelona desde el año 2006, en éste se difunden las últimas novedades y próximos lanzamientos en materia de tecnología, en un evento que reúne a cientos de marcas de dispositivos y microdispositivos, y que genera una alta expectación mediática al marcar la pauta de lo que se ofrecerá en el 
mercado. Además, reúne a los gurús de las distintas empresas tecnológicas, incluido Mark Zuckerberg (Facebook) y otros representantes de grandes compañías digitales.

En 2011, Android se hace más fuerte en lo que respecta a cuotas de mercado con una gama de móviles como Samsung, Sony Xperia y HTC, mientras que Apple lanza el iPhone 4S, que sería el primero de la línea "s" de la compañía, lo que significa que la empresa lanzaría una nueva versión con un número nuevo y al siguiente el mismo número, pero con la letra "s", esta versión representaba una mejora en el modelo anterior, ya fuera en hardware o software.

En 2012, Apple y Samsung siguen siendo los reyes del mercado, Samsung ofrece el Galaxy S3 y el Note 2, cuyos móviles contaban con funcionalidades aptas para el trabajo, mientras que Apple lanzó el iPhone 5, siendo la sexta generación de este dispositivo, uno de cuyos principales atractivos se centraba en el diseño, gracias a una pantalla más grande y a que era más delgado que su antecesor, el iPhone 4S. La carrera por la innovación no paraba, cada año las marcas sacaban nuevos productos con mejores características, enfocándose especialmente en el diseño (más delgado, más ligero), en la cámara (mejor resolución), mayor capacidad de almacenamiento, mejor procesador, mayor capacidad de batería y reproducción digital de audio y vídeo.

Desde 2013 hasta la actualidad han irrumpido en la esfera de la tecnología móvil nuevos fabricantes que han ido ganando cuota en el mercado al ofrecer dispositivos con precios más competitivos, como ya se ha mencionado anteriormente, además de casi las mismas funcionalidades al usuario. Entre las marcas más destacadas se encuentran Huawei, Xiaomi, Oppo y OnePlus. No obstante, Apple y Samsung siguen siendo referentes en el mercado y cada año lanzan un nuevo dispositivo, ya sea un teléfono móvil o una tableta. En la siguiente tabla se presentarán los modelos de teléfonos inteligentes más reconocidos por los usuarios y sus años de lanzamiento.

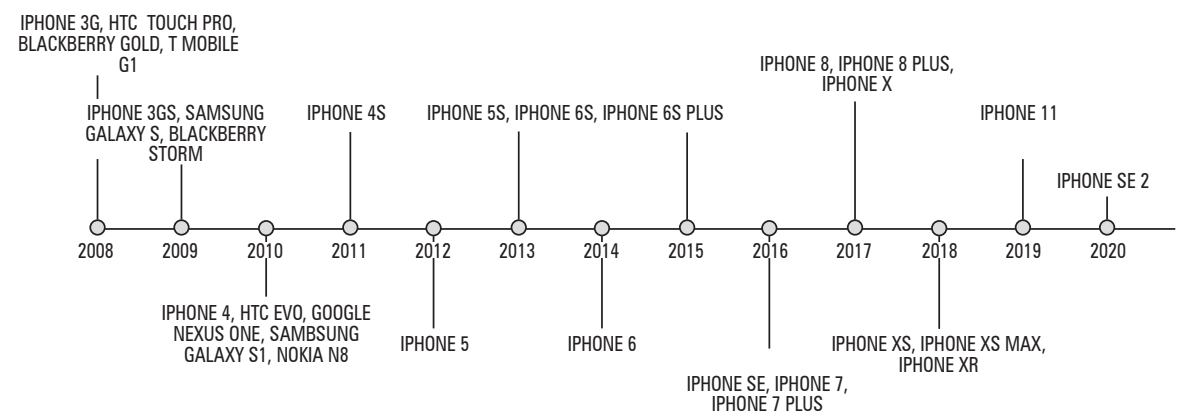

Figura 3. Principales lanzamientos de móviles inteligentes a escala mundial Fuente: elaboración propia a partir de Apple, n.d. y Samsung, n.d. 
Si bien es cierto que todos estos dispositivos van a presentar nuevas características que facilitan la comunicación y la gestión de la vida cotidiana, se hace notorio que algunas marcas hacen más de un lanzamiento cada año, incluso dos o tres, además de ofrecer otras gamas de productos de menor precio y con funcionalidades menos sofisticadas.

\section{Discusión}

Hace más de una década, entrado el siglo XXI, la gente miraba de reojo a quien tenía un teléfono móvil, un bien costoso, que representaba cierto "status" social (ejecutivo, clase alta), unos años después ese objeto costoso se popularizó, se convirtió en necesario, a medida que los avances en las redes (Internet, negocios, etc.) y junto al desarrollo de la globalización, ya no era considerado un lujo, sino que pasó a ser un objeto útil para la gestión de la vida diaria: comunicación, familia, trabajo, etc. Gerard Goggin y Hjorth (2009) y Katz y Aakhus (2002), hablan de esa conexión constante, necesaria para insertarse en el mundo actual y quedar aislado de los distintos procesos que se efectúan en la cotidianidad.

Esa alta penetración del móvil no se genera por sí misma, es decir, no es un hecho aislado, ya que llega asociado a una multitud de factores como los de una economía global (Beck, 1998), conexión constante (Katz, 2006; Katz y Aakhus, 2002), cambios en los modelos de trabajo, digitalización, comercio electrónico, entre otros. Por ello, rompiendo la visión tecnófoba sobre el hecho de que la tecnología va a dominar al ser humano, es necesario entender que estos dispositivos van a ser el medio/herramienta para que estas condiciones sociales y de mercado se generen y es así como el concepto de capitalismo tecnocientífico se relaciona con este aspecto. Un capitalismo, en el avance de la tecnología, va a ser un elemento crucial para el desarrollo de la economía y de algunos componentes sociales: educación (formación online), salud (telemedicina, divulgación), cotidianeidad (familia, conciliación, horarios, viajes, etc.) y otros que son parte de la vida de las personas. Giddens (1993), en su obra "consecuencias de la modernidad", Lipovetsky (2007) o Bauman (2006) analizaban estas nuevas formas de consumo, en su mayoría desde un punto de vista crítico, en el que se asume que el uso de tecnologías es parte de esa nueva modernidad, de un sistema capitalista digital que va a dejar al ser humano desprovisto de todo control, sin embargo, es necesario matizar que la digitalización ha traído consigo también ventajas, que le permiten al ser humano mejorar su calidad de vida y adaptarse a las distintas situaciones y, aunque no es el objeto central de este trabajo, cabe mencionar la crisis sanitaria de la COVID-19, por la que atraviesa el mundo en el 2020 y que ha puesto sobre la mesa los riesgos y las ventajas de la 
tecnología en un planeta confinado y en donde el miedo y la incertidumbre se han convertido en las sensaciones/características más destacadas del año.

\section{Conclusión}

A lo largo de este artículo se ha podido observar cómo la crisis económica que empezó desde 2008 no ha mermado el consumo tecnológico a escala global, sino todo lo contrario, los dispositivos móviles digitales llegaron para quedarse.

A la par del desarrollo tecnológico y de lo que se ha llamado en este trabajo "arqueologías" se observan no solo nuevos lanzamientos de teléfonos móviles inteligentes o de tabletas, sino también, nuevas formas de consumo tecnológico mediante el uso de plataformas de compra venta y de comercio electrónico. Pese a que se hace más marcada la brecha digital en países con bajo índice de desarrollo (International Telecommunication Union, 2018), se observa que no quedan totalmente rezagados del acceso y uso de las nuevas tecnologías, esto gracias a que los precios de estos bienes y servicios cada vez se hacen más accesibles a todas las poblaciones, debido al alto despliegue de redes y a la fabricación de dispositivos inteligentes más baratos que ayudan a que estas brechas se vayan haciendo más estrechas.

El mundo, la globalización y el capitalismo han cambiado de una manera vertiginosa al igual que la tecnología que se ha ido adaptando a esas "necesidades" y a las peticiones de consumo de los usuarios, quienes buscan nuevas innovaciones que les permitan, además, obtener más entretenimiento y acceso a contenidos multimedia.

Este nuevo capitalismo tecnocientífico se diferencia del fordismo de inicios del siglo XX (Alonso y Fernández, 2013) o del toyotismo de mediados del mismo siglo en que es mucho más dinámico y no está centrado en los objetos sino en los servicios; se ha pasado de una sociedad de productores a una de consumidores (Bauman, 2006) e incluso prosumers o prosumidores (Toffler y Martín, 1990), siendo la tecnología uno de los principales representantes, ya que se observa un consumidor más responsable, que otorga diversos usos a los bienes y servicios que adquiere, que opina, que se queja, que participa junto con el productor, no es un ser aislado, no es "ingenuo" como hace años le hacían creer. El uso de la tecnología ya no es "asunto" de jóvenes y adolescentes. Si bien es cierto que los nativos digitales (Prensky, 2001, 2010) han desarrollado mayores destrezas en el uso de dispositivos móviles y son quienes se encuentran más a la vanguardia en este aspecto, las personas de mediana edad y muchos mayores ya se han implicado en el uso de estos artefactos, lo mismo sucede con Internet, debido a que muchos países han invertido en programas de 
alfabetización digital para adultos mayores, además, de que muchos de ellos tienen como "mentores" a sus hijos y nietos que les facilitan las herramientas para que puedan usar un teléfono inteligente o una tableta. Finalmente, el móvil inteligente, como se ha visto en algunas de las estadísticas del ITU, Cepal, Eurostat y GSMA, se convierte en una herramienta multitask, una "navaja suiza" que permite realizar simultáneamente múltiples actividades, por ello, se genera una alta expectación en cada nuevo lanzamiento, ya que se adapta a lo que buscan los usuarios, siendo esa navaja cada vez más completa y dejando obsoleta a su predecesora.

\section{Referencias}

Alonso, L. E. (2004). Las políticas del consumo: transformaciones en el proceso de trabajo y fragmentación de los estilos de vida. Revista Española de Sociología, 4(1), 7-50.

Alonso, L. E. (2007). Las nuevas culturas del consumo y la sociedad fragmentada/New consumption culture and fragmented society. Pensar La Publicidad, 1(2), 13.

Alonso, L. E. y Fernández, C. J. (2013). Los discursos del presente. Un análisis de los imaginarios sociales contemporáneos. Madrid: Siglo XXI Editores.

Apple. (s.f.). iPhone. https://www.apple.com/iphone/

Baker, A. (2009). The market and the masses in Latin America: Policy reform and consumption in liberalizing economies. Cambridge University Press.

Ballesteros Doncel, E. (2016). Circulación de memes en WhatsApp: ambivalencias del humor desde la perspectiva de género. Empiria, Revista de Metodología de Ciencias Sociales, (35), 21-45. https://doi.org/10.5944/empiria.35.2016.17167

Bauman, Z. (2006). Vida de consumo. Fondo de cultura económica.

Beck, U. (1998). ¿Qué es la globalización? Falacias del globalismo, respuestas a la globalización. Paidós.

Beck, U., Lash, S. y Giddens, A. (1997). Modernización reflexiva: política, tradición y estética en el orden social moderno. Alianza Editorial.

Bermejo Berros, J. (2013). Nuevas estrategias retóricas en la sociedad de la neopublicidad. Revista Cientifica de Comunicación y Tecnologías Emergentes, 11(1), 99-124. https://doi.org/10.7195/ril4. v1li1.528

Betz, F. (2002). Strategic Business Models. Engineering Management Journal, 14(1), 21-28. https://doi. org/10.1080/10429247.2002.11415145

Boiko, I. (2019). Technological Reconstruction of the Global Economy. In Globalization. 2-22

Bouhnik, D. y Deshen, M. (2014). WhatsApp Goes to School: Mobile Instant Messaging between Teachers and Students. Journal of Information Technology Education: Research, 13(1), 217-231. https://doi.org/10.28945/2051

Bourdieu, P. (1988). La Distinción : criterios y bases sociales del gusto. Taurus. 
Bourdieu, P. (2003). Las estructuras sociales de la economía. Anagrama.

Calderón Gómez, D. (2019). Una aproximación a la evolución de la brecha digital entre la población joven en España (2006-2015). Revista Española de Sociología, 28(1), 27-44. https://doi. org/10.22325/fes/res.2018.16

Carnoy, M. y Castells, M. (2001). Globalization, the knowledge society, and the Network State: Poulantzas at the millennium. Global Networks, 1(1), 1-18.

Castells, M. (1997). La sociedad red. Vol II: El poder de a identidad. Vol III. Fin de Milenio. Alianza Editorial.

Castells, M. (2007). Comunicación móvil y sociedad: una perspectiva global. Editorial Ariel y Fundación Telefónica.

Cepal. (2014). Cepalstat: Objetivos de Desarrollo del Milenio en América Latina y El Caribe. https://cepalstat-prod.cepal.org/cepalstat/tabulador/ConsultaIntegradaProc_HTML.asp\#1

European Commision. (2019). The Digital Economy and Society Index. https://ec.europa.eu/ digital-single-market/en/digital-economy-and-society-index-desi

Eurostat. (2019). Individuals - mobile internet access. https://appsso.eurostat.ec.europa.eu/nui/ submitViewTableAction.do

Fisher, E. (2010). Media and new capitalism in the digital age: The spirit of networks. United States: Springer.

Fleming, P. (2017). The Human Capital Hoax: Work, Debt and Insecurity in the Era of Uberization. Organization Studies, 38(5), 691-709. https://doi.org/10.1177/0170840616686129

Fundación Telefónica. (2019). Sociedad Digital en España 2018. Taurus.

Gee, J. (2018). The new work order. Routledge.

Giddens, A. (1993). Consecuencias de la modernidad.Alianza Editorial.

Goggin, G. y Hjorth, L. (2009). Mobile Technologies: From Telecommunications to Media. London: Taylor y Francis. https://books.google.es/books?id=mHeQAgAAQBAJ

Goggin, G. (2006). Cell phone culture: Mobile technology in everyday life. Routledge.

Goggin, G. y Hjorth, L. (2009). The question of mobile media. In Mobile Technologies, from Telecommunications to Media. Routledge.

GSMA. (2019). La Economía Móvil, América Latina 2019. https://www.gsma.com/r/mobileeconomy/ latam-es/

International Telecommunication Union. (2018). Measuring the Information Society Report. Volume 1 . Geneva. https://www.itu.int/en/ITU-D/Statistics/Documents/publications/misr2018/MISR2018-Vol-1-E.pdf

Johnston, M. J., King, D., Arora, S., Behar, N., Athanasiou, T., Sevdalis, N. y Darzi, A. (2015) Smartphones let surgeons know WhatsApp: an analysis of communication in emergency surgical teams. The American Journal of Surgery, 209(1), 45-51. https://doi.org/10.1016/j. amjsurg.2014.08.030 
Katz, J. E. (2006). Magic in the air: Mobile communication and the transformation of social life. New Brunswick.

Katz, J. E. y Aakhus, M. (2002). Perpetual contact: Mobile communication, private talk, public performance. Cambridge University Press.

Kimble, C. y Bourdon, I. (2013). The Link Among Information Technology, Business Models, and Strategic Breakthroughs: Examples from Amazon, Dell, and eBay. Global Business and Organizational Excellence, 33(1), 58-68. https://doi.org/10.1002/joe.21523

Kotler, P. (2010). The Prosumer Movement. In H. K. Blättel-Mink B. (Ed.), Prosumer Revisited (pp. 51-60). Wiesbaden: VS Verlag für Sozialwissenschaften. https://doi.org/10.1007/978-3-531-91998-0_2

Lipovetsky, G. (2007). La felicidad paradójica: ensayo sobre la sociedad de hiperconsumo. Anagrama.

Marchetti, M. A. y Martínez, C. (2013). Un análisis del endeudamiento de las familias a partir de la encuesta del Eurosistema sobre la situación financiera y el consumo de los hogares de 2010. En Boletín Económico/Banco de España, Diciembre 2013, p. 29-40.

Márquez, R. (2006). El diseño de índices sintéticos a partir de datos secundarios: Metodologías y estrategias para el análisis social. In M. Canales Cerón (Ed.), Metodologías de la investigación social (pp. 115-140). LOM ediciones.

Martínez Carrascal, C. y Del Río, A. (2004). Las implicaciones del endeudamiento de los hogares sobre el consumo privado. Boletín Económico/Banco de España, Diciembre 2004, p. 49-54.

Mumford, L. (1969). El mito de la máquina. Emecé.

Mumford, L. (1971). Técnica y civilización (Vol. 360). Alianza Editorial.

O'Hara, K. P., Massimi, M., Harper, R., Rubens, S. y Morris, J. (2014). Everyday dwelling with WhatsApp. In Proceedings of the 17th ACM conference on Computer supported cooperative work E social computing - CSCW '14 (pp. 1131-1143). New York, USA: ACM Press. https://doi. org/10.1145/2531602.2531679

Prensky, M. (2001). Nativos digitales, inmigrantes digitales. On the Horizon, 9(5),1-7.

Prensky, M. (2010). Teaching digital natives: Partnering for real learning. Corwin Press.

Ritala, P., Golnam, A. y Wegmann, A. (2014). Coopetition-based business models: The case of Amazon.com. Industrial Marketing Management, 43(2), 236-249. https://doi.org/10.1016/j. indmarman.2013.11.005

Ritzer, G. (2000). El encanto de un mundo desencantado: revolución en los medios de consumo. Ariel.

Ritzer, G. y Jurgenson, N. (2010). Production, Consumption, Prosumption The nature of capitalism in the age of the digital 'prosumer.' Journal of Consumer Culture, 10(1), 13-36.

Rubio Romero, J. y Perlado Lamo de Espinosa, M. (2015). El fenómeno WhatsApp en el contexto de la comunicación personal: una aproximación a través de los jóvenes universitarios. Revista ICONO14 Revista Científica de Comunicación y Tecnologías Emergentes, 13(2), 73-94. https:// doi.org/10.7195/ri14.v13i2.818

Samsung. (n.d.). Mobile. 
Schiller, D. (2014). Digital depression: Information technology and economic crisis. University of Illinois Press.

Sharma, A. y Grant, D. (2011). Narrgative, drama and charismatic leadership: The case of Apple's Steve Jobs. Leadership, 7(1), 3-26. https://doi.org/10.1177/1742715010386777

Simmel, G. (2002). Sobre la aventura: ensayos filosóficos. Barcelona: Editorial Península.

Simmel, G. (1988). El individuo y la libertad. Ensayos de critica de la cultura. Peninsula, Madrid. Península.

Soengas, X., Vivar, H. y Abuín, N. (2015). Nuevas estrategias de publicidad y marketing para una sociedad hiperconectada. In A. Rodríguez de las Heras y A. Tiana (Eds.), Educación superior: Mutación digital Comunicación, Tecnología y Sociedad (pp. 115-124). Fundación Telefónica.

Toffler, A. y Martín, A. (1990). La Tercera ola. Plaza y Janés.

Tsetsi, E. y Rains, S. A. (2017). Smartphone Internet access and use: Extending the digital divide and usage gap. Mobile Media E Communication, 5(3), 239-255. https://doi. org/10.1177/2050157917708329

Yarad Jeada, V. (2017). Procesos de uso y consumo de nuevas tecnologías digitales: un análisis específico sobre las prácticas en torno a dispositivos de reproducción móvil digital. Universidad Complutense de Madrid. 